\title{
Qui est attentif aux besoins de santé de la population?
}

\author{
Max Giger, \\ membre du Comité central \\ et président de la Commission \\ pour la formation postgraduée \\ et continue de la FMH
}

1 Groupe de travail ASSM. Les futurs profils professionnels des médecins et des infirmiers dans la pratique ambulatoire et clinique. Bull Méd Suisses. 2007;88(46): 1942-52.

2 Bourgueil Y, Mousquès J, Tajahmadi A. Comment améliorer la répartition géographique des professions de santé? Les enseignements de la littérature internationale et des mesures adoptées en France. IRDES, Biblio n ${ }^{\circ} 1635$, Paris, juin 2006.

3 Steurer J. Ein Vorschlag zur Reform des Medizinstudiums. NZZ, 21. Dezember 2006.

4 Giger M: Breite Grundbildung und Vertiefung im heutigen Studium, NZZ, 21. Dezember 2006

5 Structures ambulatoires. www. gdk-cds.ch/251.0.html (accès le 11 novembre 2007).

6 Démographie médicale et réforme de la formation professionnelle des médecins. www.swtr.ch/f/ aktuell_news.html (accès le 6 novembre 2007).

7 Mathers NJ, Challis MC, Howe AC, Field NJ. Portfolios in continuing medical education - effective and efficient? Med Educ. 1999;33: 521-30.

8 www.pharmasuisse.org/de/ pharmazie_fachpublikum/03 weiterbildung/offizinpharmazie. php? navanchor $=1010086$ (accès le 11 novembre 2007).

9 Motamed S, Rougemont A. Le certificat en pharmacie communautaire et santé publique: vers un nouveau profil professionnel pour le pharmacien? Bull Méd Suisses. 2007;88(48):2045-8.
Nous ne disposons que de connaissances partielles sur les besoins de santé de la population. La recherche épidémiologique fournit des données sur la prévalence et l'incidence des maladies les plus courantes. De même, les statistiques des assureurs permettent de connaître le nombre moyen de contacts médicaux par assuré et par année. En revanche, les données relatives aux contacts de la population avec les pharmaciens ou avec d'autres spécialistes des soins de formation universitaire ou non universitaire sont lacunaires, voire complètement inexistantes, de même que les données concernant les demandes du public auprès d'institutions de conseil spécialisées et les recherches sur Internet.

Les formations prégraduée, postgraduée et continue des professions médicales et soignantes universitaires et non universitaires doivent être conçues en se référant aux besoins de santé de la population et aux patients. Ce n'est que par ce biais qu'il est possible de définir les compétences nécessaires et de formuler les objectifs et contenus de formation. Dans les réflexions relatives au regroupement des compétences dans les différentes professions, il convient de prendre en considération l'aspect des ressources financières et humaines. La continuité de la prise en charge, ce que l'on a coutume d'appeler l'itinéraire du patient, passe par une meilleure collaboration au sein de réseaux entre les différentes professions impliquées, comme par exemple entre les médecins et les infirmières et infirmiers [1]

Depuis deux ans, la nécessité de soutenir la médecine de premier recours, la pénurie de médecins dans les régions périphériques, la réorganisation des services de garde et une formation pré- et postgraduée plus efficaces sont des questions constamment débattues au niveau international [2], national [3-6] et régional. La Commission pour la formation postgraduée et continue entend mieux centrer la formation continue sur les besoins des patients et des médecins en introduisant un portfolio de formation continue [7].

Les pharmaciennes et pharmaciens en officine assurent une bonne part de la prise en charge des besoins élémentaires de santé de la population par les conseils qu'ils dispensent au patient, en lui donnant toutes les instructions nécessaires relevant de l'automédication et, lorsque cela est nécessaire, en l'orientant vers un autre intervenant. La formation des pharmaciens est réglementée par la loi sur les professions médicales (LPMéd). La formation postgraduée FPH en pharmacie d'officine s'acquiert en suivant le programme de formation postgraduée de la Société suisse des pharmaciens [8]. La formation continue est conçue pour répondre aux besoins individuels des pharmaciennes et pharmaciens et a pour objectif de mettre régulièrement à niveau les compétences des pharmaciens d'officine. Les facultés de médecine et des sciences naturelles de l'Université de Genève proposent conjointement un "Certificat en pharmacie communautaire et santé publique», un programme de formation continue «sans programme» [9], conçu précisément pour répondre aux besoins des participantes et participants. La solution adoptée pour ce programme, notamment au niveau de ses objectifs et de sa mise en œuvre, qui implique des professionnels du monde de la médecine et de la pharmacie, ne manque pas de séduire. Le programme s'insère pleinement dans le débat sur la satisfaction des besoins de santé de la population mais aussi dans celui du rôle de la médecine de premier recours: il s'agit d'appréhender les besoins de la population et d'y apporter une réponse professionnelle qui soit à la fois efficiente et soucieuse de proximité avec le patient-client. 